\title{
A Braess Type Paradox in Power Control Over Interference Channels
}

\author{
Eitan Altman*, Vijay Kamble ${ }^{\dagger}$, Hisao Kameda ${ }^{\ddagger}$ \\ * INRIA, Centre Sophia-Antipolis, 2004 Route des Lucioles, 06902 Sophia-Antipolis Cedex, France \\ $\dagger$ Department of Industrial Engineering and Management, IIT-Kharagpur, West Bengal 721302, India \\ $\ddagger$ Institute of Information Sciences and Electronics, Univ. of Tsukuba, Tsukuba, Ibaraki 305-8573, Japan
}

\begin{abstract}
The original Braess paradox has been predicted in a context of Wardrop equilibrium in a road traffic context where there is a continuum of (non-atomic) players. It was shown that the performance of all users at equilibrium becomes worse when adding a route. This paradox as well as various variants were also studied in the context of computer networks and telecommunications. We identify a new type of paradox occurring in wireless communications with some unusual properties with respect to previous models in which the paradox has been identified.
\end{abstract}

\section{INTRODUCTION}

a) Background on Braess paradox: Service providers or the network administrator may often be faced with decisions related to upgrading of the network. For example, where should one add capacity? Where should one add new links? A frequently-used heuristic approach for upgrading a network is through bottleneck analysis, where a system bottleneck is defined as "a resource or service facility whose capacity seriously limits the performance of the entire system" (see p. 13 of [14]). Bottleneck analysis consists of adding capacity to identified bottlenecks until they cease to be bottlenecks. In a non-cooperative framework, however, this heuristic approach may have devastating effects; adding capacity to a link (and in particular, to a bottleneck link) may cause delays of all users to increase; in an economic context in which users pay the service provider, this may further cause a decrease in the revenues of the provider. This problem was identified by Braess [4] in the transportation context, and has become known as the Braess paradox. See also [8], [21]. The Braess paradox has been studied as well in the context of queuing networks [3], [5], [6], [7], [9].

In the latter references both queuing delay as well as rejection probabilities were considered as performance measures. The impact of the Braess paradox on the bottleneck link in a queuing context as well as the paradoxical impact on the service provider have been studied in [19]. In all the above references, the paradoxical behavior occurs in models in which the number of users is infinitely large and the equilibrium concept is that of Wardrop equilibrium, see [23].

It has been shown, however, in [16], [17], that the problem may occur also in models involving a finite number of players (e.g. service providers) for which the Nash framework is used. The Braess paradox has further been identified and studied in the context of distributed computing [10], [11], [12] where arrivals of jobs may be routed and performed on different processors. Interestingly, in those applications, the paradox often does not occur in the context of Wardrop equilibria; see [10].

In [20] (see also [18]), it was shown that the decrease in performance due to the Braess paradox can be arbitrarily larger than the best possible network performance, but the authors showed also that the performance decrease is no more than that which occurs if twice as much traffic is routed. The result was extended and elaborated upon in more recent papers by the same authors.

An updated list of references on the Braess paradox is kept in Braess' home page at http://homepage.ruhr-uni-bo chum.de/Dietrich.Braess/\#paradox

b) Our results: We obtain a Braess paradox in the context of wireless networks. Our model builds on the possibility of a mobile to transmit information to more than one base station at a time using either macro diversity or multi-homing. We assume that interference occurs between traffic transmitted to the same base station.

c) Structure: The structure of the paper is as follows. After introducing the model in the next section, we study obtain in Section III explicit expressions for the performance obtained at symmetric equilibria. The Braess paradox and convergence issues are discussed in Section IV followed by a concluding section.

\section{MODEL}

Consider $M$ mobiles, and $b$ base stations, $B S_{j}, j=$ $1, \ldots, b$. The channel gain in terms of power from mobile $i$ to $B S_{j}$ is given by $h_{i j}$, so that a fraction $h_{i j}$ of the power transmitted by mobile $i$ over channel $j$ is actually received at base station $j$. Each base station uses another frequency. The wireless channels are considered to be the access for the mobiles to a global network and this access can be done using either one of the BSs (Base Stations). Moreover, a mobile can transmit simultaneously to several BSs. 
Mobile $i$ has a power limitation of $V_{i}$. Let mobile $i$ use a fraction $x_{i j}$ of its power $V_{i}$ to transmit to BS $j$. Define the SINR as

$$
S I N R_{i j}=\frac{h_{i j} V_{i} x_{i j}}{N_{j}+\sum_{k \neq i} h_{k j} V_{k} x_{k j}} .
$$

$N_{j}$ is the power of the thermal noise at $B S_{j}$. We assume here that the throughput achieved by user $i$ when transmitting to $B S_{j}$ is proportional to $S I N R_{i j}$. Mobile $i$ maximises the sum of its throughputs over the various base stations:

$$
\Theta_{i}=\alpha \sum_{j} S I N R_{i j}
$$

( $\alpha$ is the proportionality constant). The assumption that the throughput is linear in the SINR is often made in the literature, see e.g. [13], [15]. It does not correspond to the best achievable throughput given by the Shannon capacity $\log (1+S I N R)$. It corresponds however to the throughput obtained by various codecs as a function of the SINR. It can also be used to approximate the Shannon capacity at a regime of low SINR.

Let $x=\left(x_{1}, \ldots, x_{M}\right)$ be the vector of policies for all mobiles, where the policy for mobile $i$ is described by a $b$-dimensional vector, $x_{i}=\left(x_{i j}\right), j=1, \ldots, b$.

We seek for a Nash equilibrium, i.e. a vector of policies $x$ such that for each $i=1, \ldots, M$, and any policy $y_{i}$ for mobile $i$, we have

$$
\Theta_{i}(x) \geq \Theta_{i}\left(\left[y_{i}, x^{-i}\right]\right)
$$

In the above, $\left[y_{i}, x^{-i}\right]$ denotes the vector of policies of all mobiles obtained from $x$ by a deviation of player $i$ from $x_{i}$ to $y_{i}$.

\section{SYMMETRIC EQUILIBRIUM}

Assume that

- The number of base stations equals the number of mobiles (we denote it below by $n$ ).

- $h_{i i}$ is the same for all $i$. We shall denote it by $h_{\text {own }}$.

- $h_{i j}$ is the same for all $i$ and $j$ such that $i \neq j$; we denote it by $h_{\text {other }}$. We shall assume that without loss of generality that $h_{\text {own }} \geq h_{\text {other }}$.

- $V_{i}$ does not depend on $i$; we then omit $i$ from the notation.

- $N_{i}$ is the same for all $i$ (and the index $i$ is omitted).

First we begin by discussing the common setting of an $n$ mobile $n$ base station case. For every mobile $k$, we define a (normalized) policy as

$$
x_{k 1}, x_{k 2}, \ldots, x_{k k}, \ldots x_{k n}
$$

where

$$
x_{k 1}+x_{k 2}+\ldots+x_{k k}+\ldots+x_{k n}=1
$$

and each $0 \leq x_{k i} \leq 1$ according to which the mobiles divide their power. Thus a mobile $k$ allots power $V x_{k i}$ to a base station $i$ and so on. Thus the number of policy variables per mobile is $n-1$. Now a throughput function for a particular mobile is a function $f(k)$ of all the $n(n-$ 1) policy variables defined according to the definition of throughput mentioned earlier. Thus we get a set of throughput functions $[f(1), f(2), f(3) \ldots f(n)]$ and every mobile wants to maximize his throughput. For this set a Nash equilibrium is characterized as a set of fixed values for all $n(n-1)$ variables from which no mobile has any incentive to deviate. If this set is an interior point i.e. $0<x_{k i}<1$ for each $k, i$, this means that, assuming differentiability, at this point, the partial derivative of the throughput function for a particular mobile with respect to its (n-1) policy variables is zero for every mobile. At a boundary point where at least one $x_{k i}=1,0$, the case is a bit complex and will be considered later.

Theorem III.1. (i) The optimal throughput is obtained when each user transmits all its power on another frequency: mobile $i$ transmits all its power to base station $i$ and its throughput equals

$$
\Theta_{i}(\text { global })=\frac{\alpha V h_{\text {own }}}{N} .
$$

(ii) The optimal policy is a Nash equilibrium.

Proof.The proof is direct.

Introduce the following class $S$ of symmetric policies. Let $x=\left(x_{1}, x_{2}, x_{3}, \ldots, x_{n}\right)$ and $x_{1}+x_{2}+$ $x_{3}+\ldots+x_{n}=1$. Then mobile 1 allots $V x_{1}$ power to $B S_{1}$ and $V x_{2}, V x_{3}, \ldots, V x_{n}$ to base stations $2, \ldots, n$ respectively. Mobile $i$ allots $V x_{1}$ power to $B S_{i}$ and $V x_{2}, V x_{3}, \ldots, V x_{n}$ to base stations $i+$ $1, \ldots, n, 1,2, \ldots, i-1$ respectively.

Theorem III.2. Characterization of interior point equilibria.

(i) The policy where each mobile $i$ assigns power $z^{*}$ given by

$$
\frac{N\left(h_{\text {other }}-h_{\text {own }}\right)+h_{\text {other }}^{2} V-\frac{n-2}{n-1} h_{\text {own }} h_{\text {other }} V}{V\left(h_{\text {own }}^{2}+h_{\text {other }}^{2}\right)-\frac{n-2}{n-1} h_{\text {own }} h_{\text {other }} V}
$$

to $B S i$ and divides $V$ minus that amount equally to the other BS is a symmetric Nash equilibrium. Each player achieves the following throughput at this equilibrium:

$$
\begin{gathered}
\left.\Theta_{i} \text { (game }\right)= \\
\alpha V\left[\frac{h_{\text {own }}^{2}+h_{\text {other }}^{2}-\frac{n-2}{n-1} h_{\text {own }} h_{\text {other }}}{\left(h_{\text {own }}+\frac{h_{\text {other }}}{n-1}\right) N+h_{\text {own }} h_{\text {other }} V}\right]
\end{gathered}
$$

(iv) The aforementioned Nash equilibrium is the only Nash equilibrium among $S$ at an interior point.

Proof. Let a mobile deviate from his strategy $\left(x_{1}, x_{2}, x_{3}, \ldots, x_{n}\right)$ and adopt a distribution 
$\left(y_{1}, y_{2}, y_{3}, \ldots, y_{n}\right)$ and $y_{1}+y_{2}+y_{3}+\ldots+y_{n}=1$ where again, without loss of generality, it allots $V y_{1}$ power to $B S_{\text {own }}$. Then the throughput for this mobile equals

$$
\begin{aligned}
\Theta_{1} & =\frac{\alpha V h_{\text {own }} y_{1}}{N+V h_{\text {other }}\left(x_{2}+x_{3}+\ldots+x_{n}\right)} \\
& +\frac{\alpha V h_{\text {other }} y_{2}}{N+V h_{\text {own }} x_{1}+V h_{\text {other }}\left(x_{3}+x_{4}+x_{5} \ldots+x_{n}\right)} \\
+ & \frac{\alpha V h_{\text {other }} y_{3}}{N+V h_{\text {own }} x_{1}+V h_{\text {other }}\left(x_{2}+x_{4}+x_{5} \ldots+x_{n}\right)}+\ldots \\
+ & \frac{\alpha V h_{\text {other }} y_{n}}{N+V h_{\text {own }} x_{1}+V h_{\text {other }}\left(x_{2}+x_{3}+x_{4} \ldots+x_{n-1}\right)}
\end{aligned}
$$

which equals

$$
\begin{aligned}
\Theta_{1} & =\frac{\alpha V h_{\text {own }} y_{1}}{N+V h_{\text {other }}\left(1-x_{1}\right)} \\
+ & \frac{\alpha V h_{\text {other }} y_{2}}{N+V h_{\text {own }} x_{1}+V h_{\text {other }}\left(1-x_{1}-x_{2}\right)} \\
+ & \frac{\alpha V h_{\text {other }} y_{3}}{N+V h_{\text {own }} x_{1}+V h_{\text {other }}\left(1-x_{1}-x_{3}\right)} \\
+ & \ldots+\frac{\alpha V h_{\text {other }}\left(1-y_{1}-y_{2}-\ldots-y_{n-1}\right)}{N+V h_{\text {own }} x_{1}+V h_{\text {other }}\left(1-x_{1}-x_{n}\right)}
\end{aligned}
$$

Partially differentiating this function with respect to $y_{1}, y_{2}, y_{3}, \ldots, y_{n-1}$ and equating the differentials to zero, we get the following series of expressions

$$
\begin{gathered}
\frac{\alpha V h_{\text {own }}}{N+V h_{\text {other }}\left(1-x_{1}\right)}= \\
\frac{\alpha V h_{\text {other }}}{N+V h_{\text {own }} x_{1}+V h_{\text {other }}\left(1-x_{1}-x_{n}\right)}
\end{gathered}
$$

and

$$
\begin{gathered}
\frac{\alpha V h_{\text {other }}}{N+V h_{\text {own }} x_{1}+V h_{\text {other }}\left(1-x_{1}-x_{k}\right)}= \\
\frac{\alpha V h_{\text {other }}}{N+V h_{\text {own }} x_{1}+V h_{\text {other }}\left(1-x_{1}-x_{n}\right)}
\end{gathered}
$$

for each $k$ varying from 2 to $n-1$

From the second condition, we get

$$
x_{2}=x_{3}=\ldots=x_{n-1}=x_{n}=\frac{1-x_{1}}{n-1}
$$

which means that the power remaining after allotment to $B S_{\text {own }}$ is divided equally among the other base stations. And now, using this result in the first condition we get that $x_{1}=z^{*}$ At these values of $\left(x_{1}, x_{2}, x_{3}, \ldots, x_{n}\right)$, the mobile is indifferent. Its throughput does not depend on its policy and hence by symmetry, this policy if used by every mobile constitutes a unique Nash equilibrium among $S$ in the interior region.
So far we obtained two types of symmetrical equilibria. We can now combine interior point solutions with boundary solutions to obtain non-symmetrical equilibria.

Theorem III.3. Characterization of Nash equilibria at a boundary point.

If $n$ mobiles and $n$ base stations are split into a number of groups with no interaction between groups and each group is under an optimal or interior point symmetric Nash equilibrium, then the policies of the mobiles constitutes a Nash equilibrium for the entire problem of $n$ mobiles and $n$ base stations. Thus we can get several non symmetric Nash equilibria.

Can there be other equilibria? and in particular symmetrical ones? We provide conditions for the existence of another symmetrical equilibrium on the boundary.

Theorem III.4. (i) Consider two mobiles and two base stations. Assume that

$$
\frac{h_{\text {other }}}{N}>\frac{h_{\text {own }}}{N+V h_{\text {other }}} .
$$

Then the situation where each mobile allocates all its power to transmit to the other's base station is also an equilibrium.

(ii) At equilibrium,

$$
\Theta_{i}(\text { game })=\frac{\alpha V h_{\text {other }}}{N}
$$

Proof. If the condition of the Theorem holds and one player, say player $i$, deviates unilaterally and allots a fraction $x$ to his own base station, his throughput is

$$
\frac{\alpha V h_{\text {own }} x}{N+V h_{\text {other }}}+\frac{\alpha V h_{\text {other }}(1-x)}{N}
$$

The derivative w.r.t. $x$ is negative under the condition of the Theorem, so that mobile $i$ strictly looses in throughput by deviating.

The Theorem can be generalized to the case of $n$ mobiles and $n$ base stations. Consider a pure multi-strategy $u$ for which each mobile $i$ transmits all its power to some base station $j=b(i) \neq i$ so that the power gain for each mobile is $h_{i j}=h_{\text {other }}<h_{\text {own }}$.

Theorem III.5. (i) A necessary condition for $u$ to be an equilibrium is that $b(i)$ are different for all $i$.

(ii) Such $u$ is an equilibrium if and only if

$$
\frac{h_{\text {other }}}{N} \geq \frac{h_{\text {own }}}{N+V h_{\text {other }}(n-1) / n} .
$$

(iii) At equilibrium,

$$
\Theta_{i}(\text { game })=\frac{\alpha V h_{\text {other }}}{N}
$$




\section{PRoperties of THE INTERIOR EQUILIBRIUM}

\section{A. Braess Type Paradox}

It follows from the above Theorem that we loose the following amount of throughput at the indifference equilibrium w.r.t. the optimal solution:

$$
\Theta_{i}(\text { global })-\Theta_{i}(\text { game })=\frac{\alpha h_{\text {other }}}{N} \times \Delta
$$

where

$$
\Delta=\frac{V h_{\text {own }}^{2}+\left(h_{\text {own }}-h_{\text {other }}\right) N}{N\left(h_{\text {own }}+h_{\text {other }} /(n-1)\right)+h_{\text {own }} h_{\text {other }} V}
$$

We see that the difference vanishes if we set $h_{\text {other }}=0$ where as for any value of $h_{\text {other }}$ (assumed to be smaller than $h_{\text {own }}$ ), the difference is strictly positive. Since the globally optimal throughput does not depend on $h_{\text {other }}$ (as long as it is smaller than $h_{\text {own }}$ ), this implies that at equilibrium, the performance with $h_{\text {other }}=0$ is strictly better than with any other value of $h_{\text {other }}$. This is precisely a Braess-type paradox where each player would do better if the radio link that allows a mobile to communicate with another base station were eliminated.

This is the first case we know of an existence of Braess paradox in a problem that has several equilibria.

\section{B. Convergence}

Consider two mobiles and two base stations. Recall that

$\Theta_{1}(y, x)=\frac{\alpha V h_{\text {own }} y_{1}}{N+V h_{\text {other }}\left(1-x_{1}\right)}+\frac{\alpha V h_{\text {other }}\left(1-y_{1}\right)}{N+V h_{\text {own }} x_{1}}$

Taking the partial derivative in $y_{1}$ and in $x_{1}$ we obtain:

$$
\frac{\partial^{2} \Theta_{1}}{\partial y_{1} \partial x_{1}}=\frac{\alpha V^{2} h_{\text {other }} h_{\text {own }}}{\left(N+V h_{\text {other }}\left(1-x_{1}\right)\right)^{2}}+\frac{\alpha V^{2} h_{\text {other }} h_{\text {own }}}{\left(N+V h_{\text {own }} x_{1}\right)^{2}}
$$

which is strictly positive. By symmetry this holds also for $\Theta_{2}$. This implies that the game is super modular [1], [22], [24]. In case $x_{1}=y_{1}=0$ is not an equilibrium, then starting at these points we shall monotonically converge to an equilibrium if we use best responses to update policies.

\section{CONCLUSION}

We have identified in this paper a Braess-type paradox that occurs in the context of wireless communications. We were able to compute explicitly the degradation of performance at equilibrium as a function of the improvement of power gain. We make the following observations.

- Our analysis focused on throughput. However, if we notice that the delay needed for a mobile $i$ to transmit a unit of data is $1 / \Theta_{i}$ then we conclude that when the throughputs improves then the delay also improves (becomes smaller). Hence the paradoxical behavior in terms of throughput also occurs with respect to the delay.

- We note that the original Braess paradox deals with costs or with delays and not with throughput: the demand there is not assumed to depend on the link costs or on whether a link is added or not. In our case the paradox concerns both delay and throughput.

- In view of the Braess paradox, the natural question arises of how to upgrade a network in a way that results in an improvement of performance for all players at equilibrium. In our case

$$
\begin{gathered}
\frac{\partial \Theta_{i}(\text { game })}{\partial h_{\text {own }}}= \\
\frac{\alpha V}{\left(N h_{\text {own }} m+N h_{\text {other }}+m h_{\text {own }} h_{\text {other }} V\right)^{2}} \times \\
{\left[N\left(h_{\text {own }}^{2} m^{2}+2 m h_{\text {own }} h_{\text {other }}-h_{\text {other }}^{2}\left(m^{2}+m-1\right)\right)\right.} \\
\left.+V h_{\text {other }} m^{2}\left(h_{\text {own }}^{2}-h_{\text {other }}^{2}\right)\right]>0,
\end{gathered}
$$

where $m:=n-1$. We conclude that a way to avoid a Braess-type paradox when upgrading the network is by increasing $h_{\text {own }}$ (e.g. by using better antennas); this leads to an improvement of the throughput of all mobiles at equilibrium. Similar problems have been considered in other contexts, see [17], [2] who show that increasing capacity of direct links between a source and a destination in routing games improves the performances at equilibrium.

- As in most cases of Braess paradox (but in contrast with the paradox obtained in [10], [11], [12]) the paradox persists for the case of $n \rightarrow \infty$ (the Wardrop equilibrium).

\section{Acknowledgement}

The work of the first author was supported by the FrenchVenezuelan ECOS-Nord grant V04M01.

\section{REFERENCES}

[1] E. Altman and Z. Altman. S-modular games and power control in wireless networks. IEEE Trans. on Automatic Control, 48(5):839-842, 2003.

[2] E. Altman, R. El Azouzi, and O. Pourtallier. Avoiding paradoxes in routing games. Computer Networks, 43(2), pages 133-146, Octobre, 2003., 2003.

[3] N. G. Beans, F. P. Kelly, and P. G. Taylor. Braess's paradox in a loss network. J. Appl. Prob., 34:155-159, 1997.

[4] D. Braess. Uber ein paradoxen der werkehrsplannung. Unternehmenforschung, 12:256-268, 1968.

[5] B. Calvert, W. Solomon, and I. Ziedins. Braess's paradox in a queueing network with state-dependent routing. J. Appl. Prob., 34:134-154, 1997

[6] J. E. Cohen and C. Jeffries. Congestion resulting from increased capacity in single-server queueing networks. IEEE/ACM Trans. on Networking, 5(2):1220-1225, April 1997. 
[7] J. E. Cohen and F. P. Kelly. A paradox of congestion in a queuing network. J. Appl. Prob., 27:730-734, 1990.

[8] S. Dafermos and A. Nagurney. On some traffic equilibrium theory paradoxes. Transportation Research B, 18:101-110, 1984.

[9] H. Kameda. How harmful the paradox can be in braess/cohenkelly-jeffries networks. In IEEE INFOCOM, New York, NY, USA, June 2002.

[10] H. Kameda, E. Altman, and T. Kozawa. r case where a paradox like Braess's occurs in the Nash equilibrium but does not occur in the Wardrop equilibrium - a situation of load balancing in distributed computer systems. In Proceedings of IEEE CDC'99, Phoenix, Arizona, USA, Dec. 1999.

[11] H. Kameda, E. Altman, T. Kozawa, and Y. Hosokawa. Braess-like paradoxes in distributed computer systems. IEEE Transaction on Automatic control, 45(9):1687-1691, 2000.

[12] H. Kameda and O. Pourtallier. Paradoxes in distributed decisions on optimal load balancing for networks of homogeneous computers. Journal of the ACM, 49(3):407-433, 2002.

[13] S. L. Kim, Z. Rosberg, and J. Zander. Combined power control and transmission selection in cellular networks. In IEEE Vehicular Technology Conference, Fall 1999.

[14] H. Kobayashi. Modeling and Analysis: An Introduction to System Performance Evaluation Methodology. Addison Wesley, 1978.

[15] I. Koo, J. Ahn, H. A. Lee, and K. Kim. Analysis of Erlang capacity for the multimedia DS-CDMA systems. IEICE Trans. Fundamentals, E82-A(5):849-855, May 1999.

[16] Y. A. Korilis, A. A. Lazar, and A. Orda. Architecting noncooperative networks. J. on Selected Areas in Communications, 13(7):1241-1251, Sept. 1995.

[17] Y. A. Korilis, A. A. Lazar, and A. Orda. Avoiding the Braess paradox in non-cooperative networks. Journal of Applied Probability, 36:211-222, 1999.

[18] E. Koutsoupias and C. Papadimitriou. Worst-case equilibria. In 16th Annual Symposium on Theoretical Aspects of Computer Science, pages 404-413, Trier, Germany, March 1999.

[19] Y. Masuda. Capacity management in decentralized networks. $m s$, 48:1628-1634, 2002.

[20] T. Roughgarden and É. Tardos. How bad is selfish routing? Journal of the ACM, 49:236-259, 2002.

[21] M. J. Smith. In a road network, increasing delay locally can reduce delay globally. Transportation Res., 12:419-422, 1978.

[22] D. Topkis. Equilibrium points in nonzero-sum n-person submodular games. SIAM J. Control and Optimization, 17:773-787, Nov. 1979.

[23] J. G. Wardrop. Some theoretical aspects of road traffic research communication networks. Proc. Inst. Civ. Eng., Part 2, 1:325378, 1952.

[24] D. D. Yao. S-modular games with queueing applications. Queueing Systems, 21:449-475, 1995. 